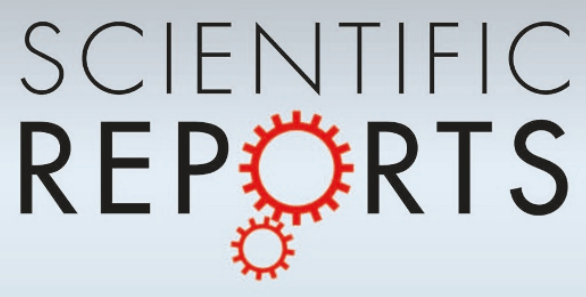

OPEN

SUBJECT AREAS:

MOLECULAR BIOLOGY

CHROMATIN

CHROMATIN STRUCTURE

CHROMATIN REMODELLING

Received

10 May 2013

Accepted

10 January 2014

Published

29 January 2014

Correspondence and requests for materials should be addressed to S.H.A. (SajadHamid.

Ahanger@usz.ch)

* Current address:

Manipal Institute of

Regenerative

Medicine, Bangalore

560065 , India.

\section{Ectopically tethered CP190 induces large-scale chromatin decondensation}

\author{
Sajad H. Ahanger ${ }^{1,2}$, Katharina Günther ${ }^{2}$, Oliver Weth ${ }^{2}$, Marek Bartkuhn $^{2}$, Ramesh R. Bhonde ${ }^{1 *}$, \\ Yogesh S. Shouche' \& Rainer Renkawitz ${ }^{2}$
}

${ }^{1}$ National Centre for Cell Science, Pune 41 1007, India, ${ }^{2}$ Institute for Genetics, Justus-Liebig University, Giessen D-35392, Germany.

Insulator mediated alteration in higher-order chromatin and/or nucleosome organization is an important aspect of epigenetic gene regulation. Recent studies have suggested a key role for CP190 in such processes. In this study, we analysed the effects of ectopically tethered insulator factors on chromatin structure and found that CP190 induces large-scale decondensation when targeted to a condensed lacO array in mammalian and Drosophila cells. In contrast, dCTCF alone, is unable to cause such a decondensation, however, when CP190 is present, $\mathrm{dCTCF}$ recruits it to the lacO array and mediates chromatin unfolding. The CP190 induced opening of chromatin may not be correlated with transcriptional activation, as binding of CP190 does not enhance luciferase activity in reporter assays. We propose that CP190 may mediate histone modification and chromatin remodelling activity to induce an open chromatin state by its direct recruitment or targeting by a DNA binding factor such as dCTCF.

T he packaging of eukaryotic DNA into nucleosomes and its subsequent folding into higher-order chromatin has a direct effect on regulation of gene expression. Chromatin insulators or boundaries are key to this organization across the species. These elements along with their associated proteins interact with each other or with other regulatory factors to divide the genome into functionally autonomous units ${ }^{1-3}$. In vertebrates, CTCF remains the major insulator factor, although the presence of vertebrate GAF has been reported ${ }^{4,5}$. The Drosophila genome contains the most diverse insulators reported so far. There are at least five insulator binding proteins that have been studied in detail. These include Zeste-White 5 (Zw5), Boundary Element Associated Factor 32 (BEAF32), the GAGA factor (GAF), Suppressor of Hairy-wing $\{\mathrm{Su}(\mathrm{Hw})\}$ and CCCTC binding factor $(\mathrm{dCTCF})^{6-10}$. In addition to the above factors, all the insulators share Centrosomal Protein 190 (CP190) and one of the $\operatorname{Mod}(\mathrm{mdg} 4)$ isoforms as co-factors ${ }^{11}$. Despite the fact that several insulators have been identified, very little is known about their mechanism of action. It has been suggested that insulators are involved in organising higherorder chromatin structure via long distance interactions and looping of chromosomal regions ${ }^{12}$. Insulators can also directly interact with the transcriptional machinery to interfere with communication between regulatory elements and promoters ${ }^{13,14}$.

Several studies have suggested that insulators may function via remodelling of chromatin structure ${ }^{15-17}$. Changes in chromatin structure are required to allow accessibility to regulatory factors and enzymatic complexes that are needed to accommodate various nuclear functions. Such modifications are brought about by changes in higher-order chromatin structure or movement, alteration or removal of nucleosomes ${ }^{18}$. In vertebrates, CTCF has been associated with well positioned nucleosomes ${ }^{19-21}$ and it is suggested that positioning of nucleosomes and chromatin remodelling is an important component of CTCF function ${ }^{20}$. The CTCF bound sites in Drosophila show a regular nucleosomal occupancy, but interestingly, CTCF sites that are co-bound by CP190 show a prominent dip in nucleosome occupancy/or high histone replacement and mark the boundaries of H3K27me3 domains ${ }^{22,23}$. The nucleosome depletion at dCTCF/CP190 bound regions has been shown to depend on CP190 alone ${ }^{22}$. These studies indicate that alteration of chromatin structure induced by insulator factors may play an important role in setting up the boundary function. To further understand the influence of insulator factors on chromatin organization, we tested the effects of ectopically tethered dCTCF and CP190 on higherorder chromatin using the lacO-LacI tethering system ${ }^{24}$. We found that upon tethering to the condensed lacO array, CP190 induces large-scale chromatin decondensation in mammalian and Drosophila cells. Drosophila CTCF (dCTCF), on the other hand, does not induce such a change in chromatin structure, "however, when CP190 is present, dCTCF recruits it to the lacO array and mediates unfolding of the chromatin. Based on these results, we suggest that modulation of chromatin structure is an important aspect of CP190 dependent insulator function in Drosophila and probably in other insects too. 


\section{Results}

Ectopically tethered CP190 induces decondensation of chromatin at the lacO array in mammalian cells. To examine the effects of insulator factors on higher-order chromatin structure, we used lac operator-repressor (lacO-LacI) tethering system ${ }^{24}$. The lacO-LacI system contains two components; a construct expressing lac repressor DNA binding domain (LacI) fused in frame to insulator proteins, and $\mathrm{U} 2 \mathrm{OS}$ cell clone $\mathrm{F} 42 \mathrm{~B} 8^{25}$ having repetitive binding sites for LacI ( $\mathrm{lacO}$ ) integrated at the heterochromatic regions. All the fusion constructs are tagged with GFP to easily monitor changes in the highly condensed lacO array, following tethering of insulator factors. The expression of insulator fusion constructs was verified by western blotting using anti-dCTCF, anti-CP190 and anti-GFP antibodies (Supplementary figure $\mathrm{S} 1$ and $\mathrm{S} 2$ ). The single lacO repeat cluster was identified by specific binding of GFP-LacI and cherry-LacI both of which mark the same spot (Supplementary figure S3a). Furthermore, U2OS cells in the absence of an integrated $\mathrm{LacO}$ repeat cluster are not marked by a single spot after expression of the LacI-fusion constructs (Supplementary figure S3b). We transiently transfected F42B8 cells with GFP-LacI, GFP-LacI fused to chCTCF, dCTCF or CP190 and analysed the a

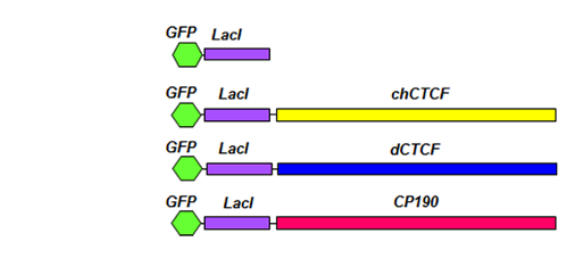

b

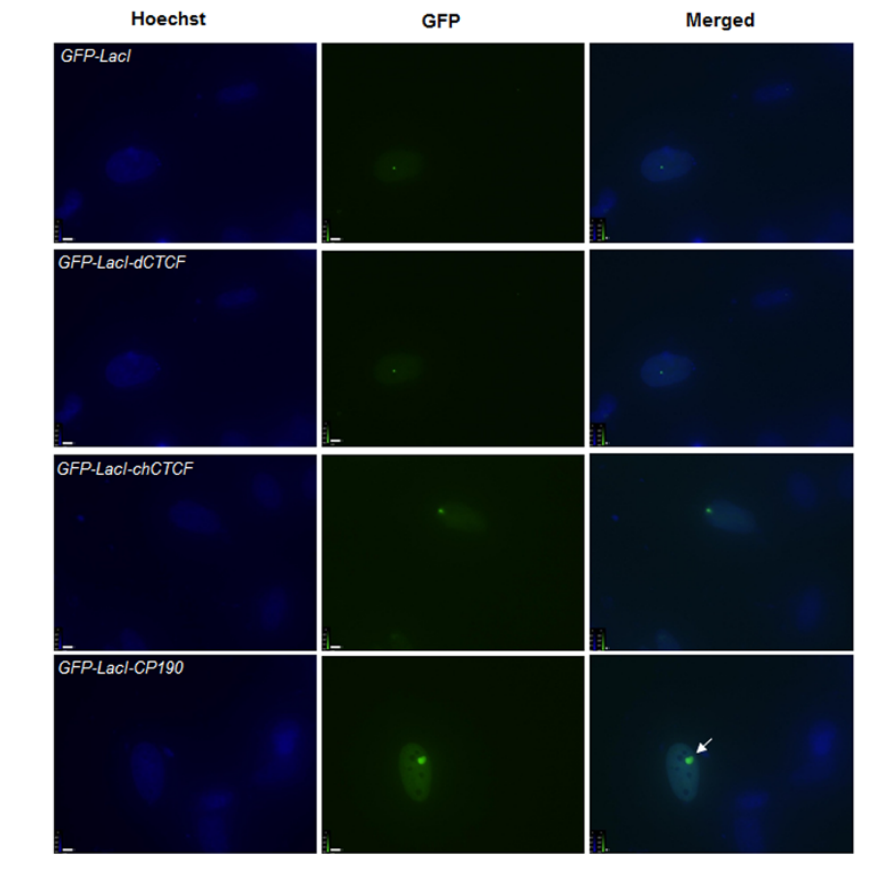

changes in chromatin structure after 48 hours of transfection by fluorescence microscopy (Figure 1a, b). Chicken CTCF was used as a positive control, since it is very similar to human CTCF but can be distinguished from endogenous CTCF. Targeting of GFP-LacI (which serves as a negative control) to the lac operator array did not change the condensed state of the locus, as it was still seen as a small round dot (Figure 1b). In contrast, upon tethering of insulator factors to the condensed lacO array, we observed that vertebrate CTCF (chicken CTCF) which is known to cause chromatin decondensation of the lacO repeat ${ }^{26}$ expands the array, whereas Drosophila CTCF (dCTCF) does not (Figure 1b). Interestingly, tethering CP190 to the lacO array resulted in transformation of the array from a condensed small dot to a large ball-shaped appearance, indicating a strong decondensation of the local chromatin (Figure 1b). The chromatin decondensation induced by CP190 was robust and consistent as more than $90 \%$ of the transfected cells showed decondensation of the lacO array.

In Drosophila, the insulator function of CTCF is often dependent on CP190 27,28 . We wondered whether the inability of GFP-LacIdCTCF to mediate decondensation of the lacO array was due to the absence of CP190 in vertebrate (F42B8) cells. To test this, we c

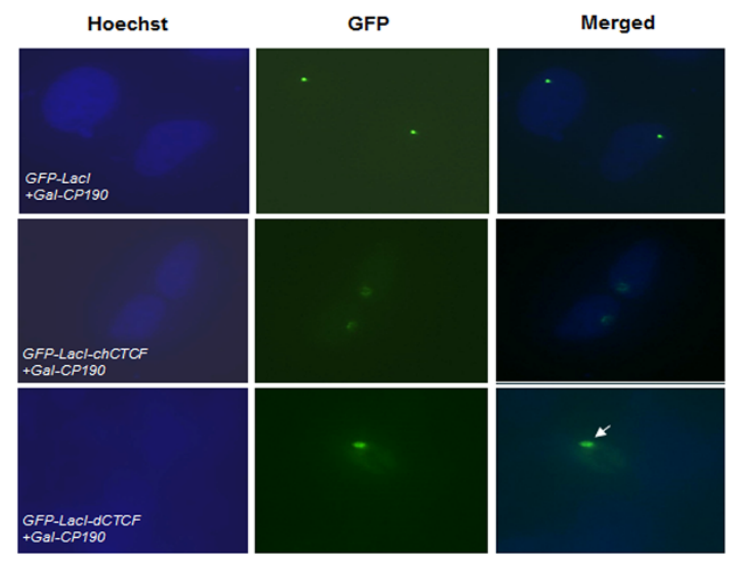

d

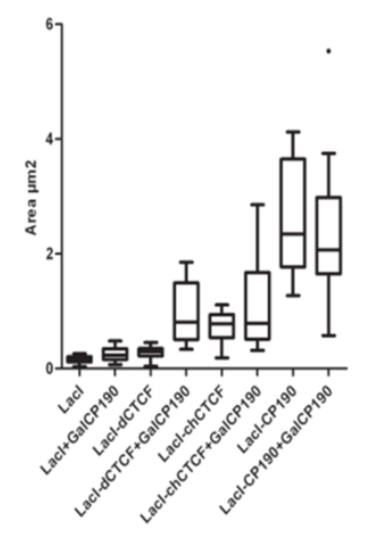

Figure $1 \mid$ Ectopically tethered CP190 induces large-scale chromatin decondensation at the lacO array in mammalian cells. (a). GFP-LacI-insulator fusion constructs. (b). The F42B8 cell clone of U2OS cells with a LacO repeat cluster integrated close to the centromere, which constitutes a heterochromatic domain, were transiently transfected with GFP-LacI, or GFP-LacI fused to chCTCF, dCTCF or CP190. Cells were fixed and stained with Hoechst stain and observed under fluorescence microscopy. (c). F42B8 cells were transfected with GFP-LacI, GFP-LacI-chCTCF, or GFP-LacI-dCTCF alone or with Gal4-CP190 and analyzed as above. Note that GFP-LacI-dCTCF is able induce decondensation in presence of Gal4-CP190 (indicated by the white arrow). (d). Quantitative analysis of the decondensation of the lacO array (size of the array in $\mu \mathrm{m}^{2}$ ) upon targeting of insulator fusion proteins measured by the Volocity software. 
used a construct expressing Gal4-CP190 (lacking GFP-LacI tethering domain) and verified its expression by western blotting (Supplementary figure S4). F42B8 cells were co-transfected with GFPLacI-dCTCF and Gal4-CP190 and analysed for changes in chromatin structure at the lacO array. For comparison, cells were also co-transfected with Gal4-CP190 and GFP-LacI-chCTCF or GFP-LacI. There was no change in the condensed lacO array when GFP-LacI was transfected alone or with Gal4-CP190 (Figure 1b and c, top panel). A mild increase in decondensation was observed when GFP-LacIchCTCF was transfected with Gal4-CP190 (Figure 1b and c, middle panel, for quantitative evaluation see below and figure 1d). Interestingly, cells co-transfected with GFP-LacI-dCTCF and Gal4CP190 showed a significant decondensation of the lacO array when compared to cells transfected with GFP-LacI-dCTCF alone (Figure 1b and c, bottom panel) suggesting that dCTCF needs CP190 to open up the lacO chromatin.

To quantify the extent of decondensation, we measured the size of the lacO array upon targeting of chCTCF, dCTCF and CP190 alone or with Gal4-CP190 (Supplementary table 1a). 20 nuclei were measured for changes in the area of the array and an average was presented as box-plot (Figure 1d). Upon CP190 targeting (LacICP190), the average area of the spot is more than 15 fold larger than targeting GFP-LacI. LacI-chCTCF induced decondensation of the array is not significantly extended by the addition of Gal4CP190. Furthermore, the array size, as seen with GFP-LacI-dCTCF is about threefold increased upon co-expression of Gal4-CP190. These results suggest that targeting CP190 to a defined chromatin locus either by direct LacI mediated DNA binding or by the less efficient recruitment to DNA bound LacI-dCTCF is able to induce chromatin decondensation in that region. This suggestion is further analysed below.

GFP-LacI-dCTCF recruits CP190 to lacO array and induces decondensation. Recent genome-wide studies have shown that majority of the binding sites of dCTCF are co- occupied by CP19022,29. Additionally, CP190 has been shown to interact with $\mathrm{dCTCF}$ and is required for its insulator function ${ }^{27,28}$. Similar results have been achieved with other DNA binding insulator factors of Drosophila, which all target CP190 to chromatin ${ }^{11}$. These studies suggest that CP190 is recruited to its target sites by insulator binding proteins such as dCTCF. Therefore, we reasoned that unfolding of chromatin at the lacO array observed upon cotransfection of GFP-LacI-dCTCF and Gal4-CP190 is mediated through the recruitment of latter by the former. To test our hypothesis, we analysed GFP-LacI-dCTCF and Gal4-CP190 cotransfected cells by immuno-staining using anti-CP190 antibody. For comparison, cells co-transfected with Gal4-CP190 and GFPLacI-chCTCF or GFP-LacI were also analysed. Cells transfected with GFP-LacI-CP190 were used as a positive control for immunostaining. We could clearly see the recruitment of Gal4CP190 to lacO array in those cells, which were co-transfected with GFP-LacI-dCTCF, and not in the cells co-transfected with GFPLacI-chCTCF or GFP-LacI (Figure 2). Moreover, the lacO array was decondensed in only those cells, which showed recruitment of
GFP
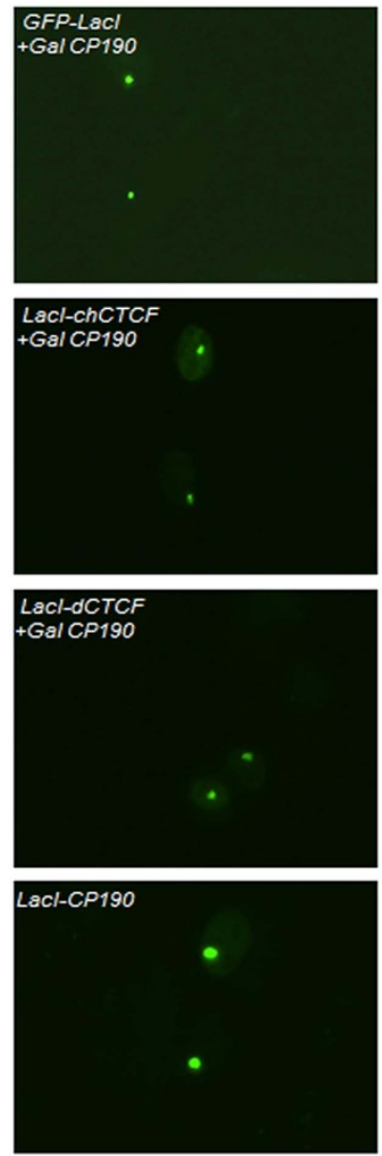

a-CP190
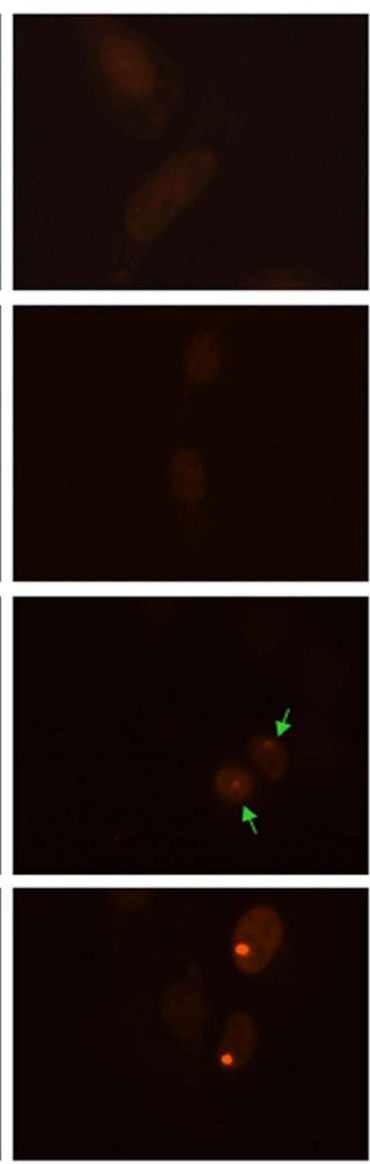

Merged
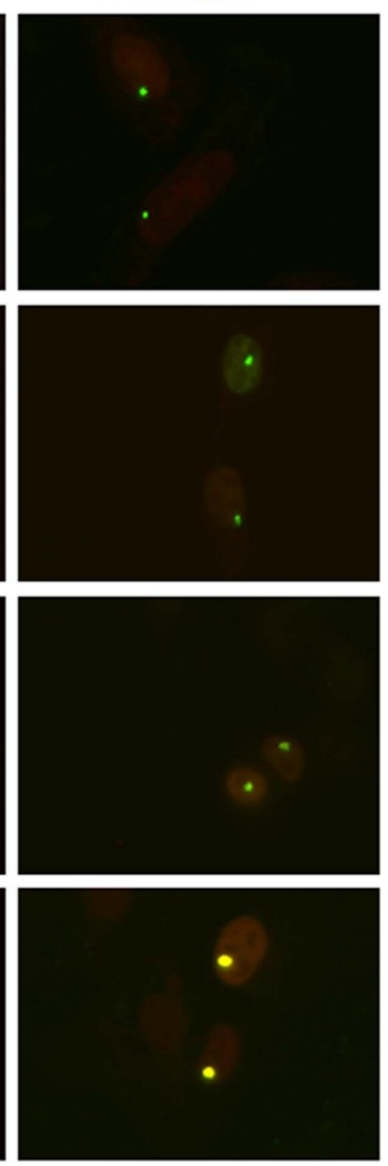

Figure $2 \mid$ GFP-LacI-dCTCF induces decondensation of the lacO array by recruiting Gal4-CP190. F42B8 cells were transfected as above, with Gal4CP190 and GFP-LacI, GFP-LacI-chCTCF, or GFP-LacI-dCTCF. Cells were fixed post 48 hours of transfection and analysed by immuno-staining using anti-CP190 antibody (Bx63). As seen in panel 3, only LacI-dCTCF is able to recruit CP190 to the lacO array (shown by green arrows). LacI-CP190 (bottom panel) was used as a positive control for staining. 
GFP
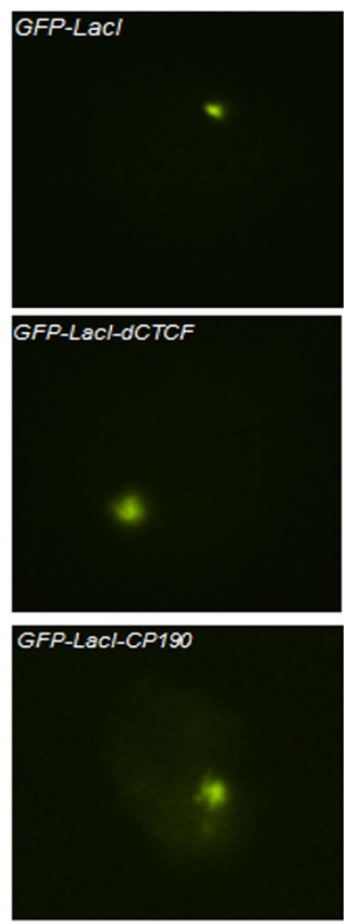

Merged
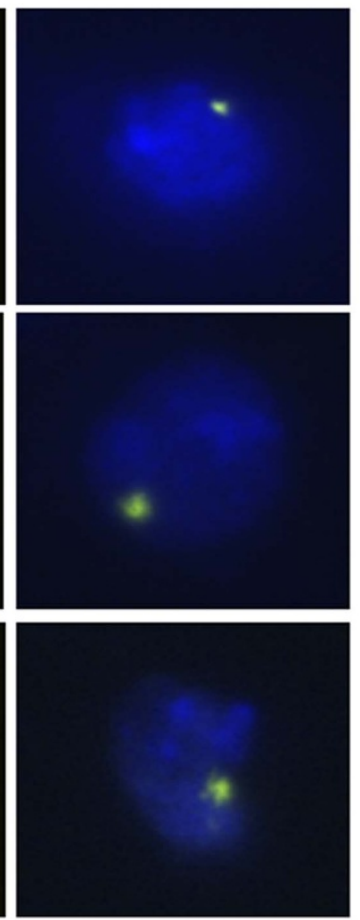

b

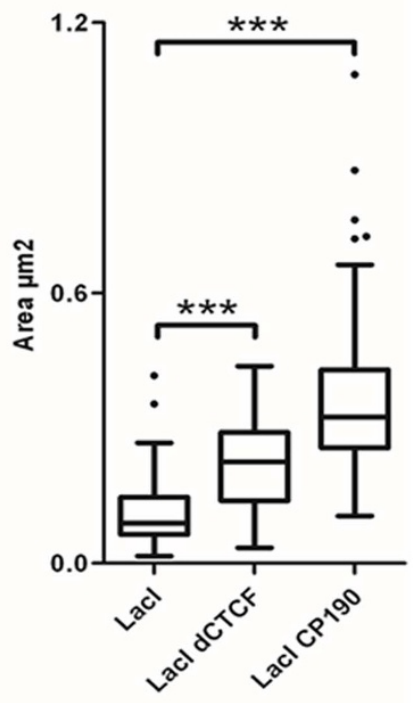

Figure 3 dCTCF and CP190 result in decondensation of an integrated LacO array in S2 cells. S2 lacO cells were transfected with GFP-LacI, GFP-LacIdCTCF and GFP-LacI-CP190. Induction of the metallothionein promoter (pMT vectors) was performed by addition of $500 \mu \mathrm{M} \mathrm{CuSO}_{4}$ for 24 hrs. (a). The cells were fixed and stained with Hoechst to visualize the nucleus. The left panels show the GFP tagged proteins and the right show the merge with Hoechst. (b). The size (in $\left.\mu \mathrm{m}^{2}\right)$ of representative arrays $(\sim 50)$ was measured by the Volocity software and presented as a box plot, whereas whiskers are defined according to Tukey. Significance was controlled with a two tailed Mann-Whitney-test (*** $\mathrm{p} \leq 0.001)$.

CP190. These results suggest that only dCTCF is able to interact with CP190 and recruit it to the lacO array to mediate chromatin decondensation. Taken together, our results imply that CP190 can bring about chromatin decondensation either through its direct recruitment (in this case via LacI tethering domain) to its target loci or recruitment facilitated by a DNA binding factor such as dCTCF.

Targeting CP190 to lacO array in Drosophila cells also leads to chromatin decondensation. To examine the effect of CP190 on chromatin decondensation in a more natural context, we used Drosophila S2 cells containing an autosomal integration of the lacO array (a kind gift from Patrick Heun) ${ }^{30}$. S2-lacO cells were transfected with GFP-LacI, GFP-LacI-dCTCF or GFP-LacI-CP190. These fusion proteins are under the control of the metallothionein promoter and expression was induced by $\mathrm{CuSO}_{4}$. Comparable to the results obtained with F42B8 cells, targeting CP190 to the S2-lacO array induced a strong decondensation of the local chromatin (Figure 3). However, in contrast to F42B8 cells, GFP-LacI-dCTCF itself is also able to expand the condensed S2-lacO array. This very likely is mediated by the ubiquitous Drosophila factor CP190, which is present in S2 cells and therefore will be recruited to lacO bound GFP-LacI-dCTCF (Figure 3). As control, targeting GFP-LacI does not induce any change in the condensed S2-lacO array. As expected and as observed in F42B8 cells, the extent of decondensation induced by GFP-LacI-CP190 is much stronger than GFP-LacI-dCTCF, since the latter requires recruitment of endogenous CP190, whereas the former binds to the lacO array directly and with high affinity (Figure 3b, Supplementary table 1b). Overall, these results confirm our previous observations in mammalian F42B8 cells and suggest that CP190 is sufficient to induce strong decondensation of the chromatin in its natural environment, however, it needs a recruiting factor to execute this function.

Ectopically tethered CP190 does not transactivate luciferase in transient reporter assay. To study if there is any correlation between opening of chromatin by GFP-LacI-CP190 and gene activity we used transient luciferase assay in HEK 293T cells. The assay system contains a vector having seven repeats of lacO binding site (7×lacO) upstream of the SV40-promoter driving the expression of luciferase ${ }^{31}$. The lacO recognition sequences provides the binding site for LacI-insulator fusion proteins which can be easily used to test the effects of LacI fused insulator constructs on luciferase activity (Figure 4). HEK293T cells were transiently co-transfected with pGL3-Luc-7lacO and GFP-LacI-CP190 using calcium phosphate method. Cells were harvested after 24 hours and luciferase activity was measured in a Luminometer. Lac- $Z$ was used for normalization. For comparisons, cells were also transfected with GFP-LacI-dCTCF, GFP-LacI-chCTCF and GFP-LacI. Expression of GFP-LacI-CP190 did not result in significant changes in luciferase activity when compared to empty vector (pGL3-luc-7lacO) and GFP-LacI controls (Figure 4). In contrast, expression of dCTCF and chCTCF LacI fusion constructs led to reduction in luciferase activity (Figure 4). These results suggest that chromatin decondensation mediated by LacI-CP190 may not have a direct influence on transcriptional activity.

Overall, our results suggest that opening of chromatin structure may be a key aspect of CP190 function in Drosophila and probably in other insects too and that CP190 may mediate chromatin remodelling activity through its interaction and recruitment of chromatin remodelling machinery to its target loci. 
$7 \times \mathrm{LacO}$ pSV 40
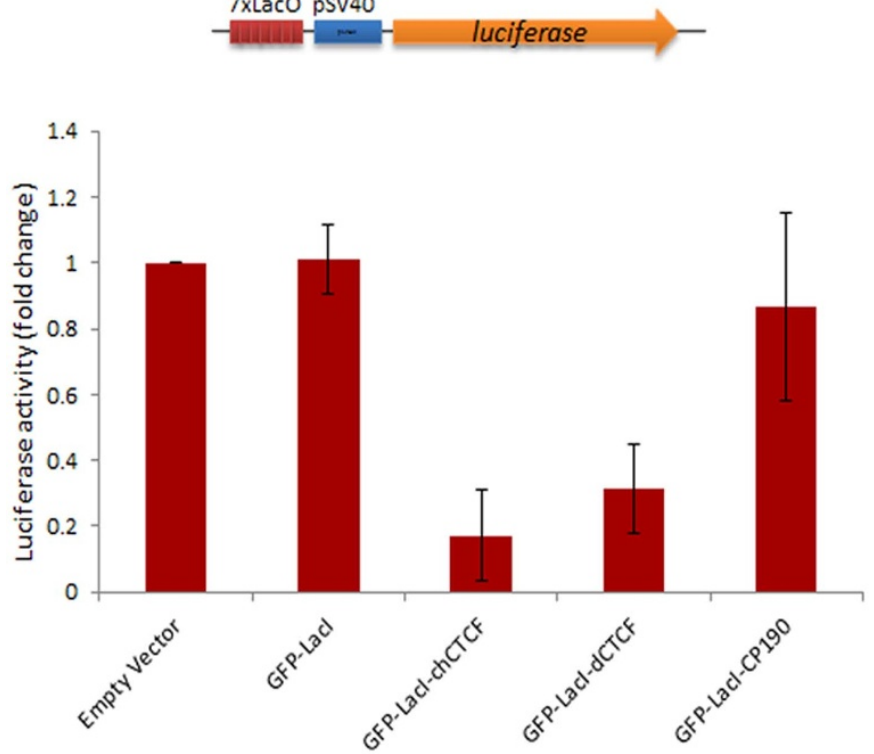

Figure $4 \mid$ Tethering of CP190 does not lead to increased luciferase activity. HEK 293T cells were transiently transfected with pGL3-luc-7lacO reporter (shown on top) and the indicated LacI fusion constructs. After 24 hours of transfection, the cells were harvested and lysed to measure luciferase activity relative to beta- galactosidase activity. Bars represent fold change luciferase activity of insulator-LacI fusion constructs upon targeting to $7 \times$ lacO repeat. Values are the mean standard error of two independent experiments.

\section{Discussion}

Modulation of chromatin structure is an important aspect of epigenetic gene regulation. Insulators are implicated in organizing chromatin into functionally separate entities through the formation of chromatin loops and CP190 is emerging as an important player in mediating such processes ${ }^{11,12,32}$. Insulators are also associated with chromatin accessibility through their interaction with chromatin remodelling machinery to alter primary or higher level of chromatin structure in their vicinity ${ }^{13}$. In order to study insulator associated changes in higher-chromatin structure, we used the lac operatorrepressor system ${ }^{33}$. This system has been widely used to study effects of chromatin binding factors on higher order-chromatin structure $^{26,34-38}$. Using this system, we could show that targeting CP190 to the condensed lacO array in mammalian and Drosophila cells induces large-scale unfolding of the local chromatin structure. We also show that dCTCF, which on its own is unable to induce chromatin unfolding, mediates decondensation of the lacO array by targeting CP190. These results suggest that CP190 might function by inducing changes in higher-order chromatin structure, however, it might need another insulator factor such as dCTCF for its targeting. This is supported by the fact that although, CP190 is essential for the insulator function in Drosophila, recruitment to its target sites is often dependent on other insulator binding proteins, such as SU(HW), BEAF-32, ZW5 or yet unknown DNA binding factors ${ }^{39}$. We also observed that binding of CP190 to lacO repeats upstream of the luciferase reporter has no effect on its transcription, although binding of dCTCF and chCTCF repress expression. CTCF is known to act both as an activator and a repressor in Drosophila as well as in vertebrates ${ }^{40}$. Our results indicate that, while in vertebrates, CTCF might carry out multiple functions of repression, activation and chromatin opening, in Drosophila the latter function can be assigned to CP190 without having a direct influence on transcription.

The underlying mechanisms of CP190 mediated changes in higher-order chromatin structure and their biological significance are difficult questions to be answered and need further investigation.
However, several mechanisms could be proposed. Recruitment of histone acetyltransferases and hyperacetylation of histones could be one mechanism. Generally, acetylation of histone $\mathrm{H} 3$ and $\mathrm{H} 4$ and the methylation of histone $\mathrm{H} 3$ on lys 4 are associated with active chromatin domains, while methylation of histone $\mathrm{H} 3$ at lys9 is usually correlated with heterochromatin ${ }^{41,42}$. Recruitment of HATs such as GCN5, P/CAF and CBP/P300 and hyperacetylation has been shown to occur at the lacO array upon VP16 tethering ${ }^{37}$. Interestingly, insulator factors have also been shown to recruit histone modifications. For example, the cHS4 insulator binding proteins USF recruits histone acetyltransferases (HATs) PCAF and P300/CBP and methyltransferase SET7/9 ${ }^{43}$. HATs and SET7/9 methyltransferase recruited by USF marks $\mathrm{H} 3$ and $\mathrm{H} 4$ acetylation and $\mathrm{H} 3$ lysine 4 methylation to form a histone hyperacetylation- and H3 lysine 4 methylation-enriched cHS4 boundary to block the spread of neighboring heterochromatin ${ }^{43}$. Another mechanism of large-scale decondensation may involve chromatin remodelling machineries. Previous studies have revealed that chromatin barriers in yeast and vertebrates utilize chromatin-remodeling factors to establish centers of open chromatin structure that impede the spread of silent chromatin ${ }^{43-45}$. These reports suggest that insulator factors indeed bring about changes in higher order chromatin structure that involve histone modification associated with open chromatin structure. However, changes in chromatin structure may not always involve hyperacetylation and chromatin remodeling factors. It has been demonstrated that targeting of UBF to the lacO array mediates large-scale chromatin decondensation but does not involve hyperacetylation of histones or chromatin remodelling complexes. Instead, UBF recruits PolI specific, TATA box-binding protein containing complex SL1 and PolI subunit ${ }^{34}$. Therefore, several possibilities need to be explored to understand CP190 mediated changes in higherorder chromatin unfolding.

Insulator factors have also been implicated in changes that occur at the primary/or nucleosomal level of chromatin. Previous studies have shown a negative correlation between insulators and nucleosome occupancy ${ }^{19,23,46,47}$ and it has been suggested that insulator factors such as CTCF may possess chromatin remodelling features ${ }^{19,20}$. In Drosophila, most of the CTCF target sites (as well as target sites of other insulator binding factors) are co-occupied by $\mathrm{CP} 190^{23,29}$. Sites that are co-bound by dCTCF/CP190 show low nucleosome occupancy, whereas sites bound by dCTCF alone show a regular nucleosomal occupancy ${ }^{22}$. Similarly, depletion of CP190 restores the dip in nucleosome occupancy at these regions suggesting that CP190 is the key player in disrupting the nucleosome organization ${ }^{22}$. It is interesting to note that while vertebrate CTCF is able to induce an open chromatin state, Drosophila CTCF appears to have lost this property and instead this function is taken over by CP190. We suggest that CP190 may indeed be a key player in the formation of open chromatin structure and preventing the spread of heterochromatin through its co-operation with insulator binding factors and remodelling machineries.

We may suggest that despite the similarity of the eleven zinc-finger DNA binding domain of vertebrate and Drosophila CTCF, there is a major difference in insulator function. In case of vertebrate CTCF, chromatin remodelling complexes and/or histone modifying enzymes might be directly recruited by CTCF. In Drosophila, several DNA binding insulator factors are known, which all bind CP190. Rather having all five insulator factors mediating chromatin opening, the unifying CP190 factor may recruit the required chromatin modifying enzymes.

\section{Methods}

Construction of GFP-lacI-insulator fusion transgenes. A linker with Acc65I (Linker Oligo-F-5' GTACCCCTCCTAAGAAGAAGAGGAAGGTTGCCCGGGATCCACAGATCTTTAAGCTTTCCTCGAGT 3') and BamHI (Linker Oligo-R-5 GATCACTCGAGGAAAGCTTAAAGATCTGTGGATCCCGGGCAACCTTCCTCTTCTTCTTAGGAGGG 3') overhangs was inserted into pSV-GFP-LacI 
(hereafter called as GFP-LacI) to incorporate few additional restriction sites for subsequent cloning of dCTCF and CP190. dCTCF was cut out from pBSK-dCTCF as BamH I fragment and cloned into Bgl II of GFP-LacI (now called as GFP-LacIdCTCF). CP190 was taken out from pAB-Gal4-CP190 and cloned as Hind III fragment into GFP-LacI (now referred to as GFP-LacI-CP190). GFP-LacI fused to chicken CTCF (GFP-LacI-chCTCF) was a gift from lab colleague Christine P. The vector pSV2 GFP-LacI-chCTCF was generated by removing a stop-codon in front of the MCS from the plasmid pSV2 GFP-LacI ${ }^{25}$ followed by SmaI digestion. The coding sequence for chCTCF was excised with EcoR1 from pEGFP-C2-CTCF ${ }^{48}$ blunt ended and inserted into the vector backbone. Gal4-CP190 was generated by cloning CP190 coding region in frame behind the Gal94 region of the vector $\mathrm{pAB}-\mathrm{Gal} 94^{49}$. $\mathrm{pMT}$ GFP-LacI-dCTCF was constructed by cutting pSV-LacI-dCTCF with EcoRV and XhoI (partial) and ligating the corresponding fragment into pMT-GFP-LacI ${ }^{30}$ digested with EcoRV and SalI. The plasmid pMT-GFP-LacI-CP190 was cloned by cutting pSV-LacI-CP190 with EcoRV and XhoI. Ligation was performed with the same strategy.

Verification of the constructs by western blotting. HEK 293T cells were transiently transfected with the GFP-LacI-CP190 and GFP-LacI-dCTCF by Calcium Phosphate method. Cells were harvested after 24 hours of transfection and a cell lysate was prepared. The protein concentration was determined by Bradford method and equal amounts of protein from each transfected construct was run on an SDS-PAGE, transferred by wet blotting to a PVDF membrane and probed with anti-dCTCF antiCP190 and anti-GFP antibodies.

Cell culture, transfections and luciferase assay. The F42B8 cell clone of U2OS cells with a LacO repeat cluster integrated close to the centromere ${ }^{25}$ and HEK 293T cells were cultured at $37^{\circ} \mathrm{C}\left(5 \% \mathrm{CO}_{2}\right)$ in Dulbecco's Modified Eagel Medium (DMEM) containing $10 \%$ fetal bovine serum. Transfections were done by JetPIE reagent (Polyplus) following manufacturer's instructions. Briefly, $2 \times 10^{5}$ cells were seeded per six-well plate and grown for 24 hours before transfection. $1 \mu \mathrm{g}$ of GFP-LacIinsulator fusion construct was transfected in F42B8 cell clone of U2OS cells. Media was replaced five hours post transfection and cells were continued to grow for 48 hours. Cells were rinsed with phosphate-buffered saline (PBS), fixed in $4 \%$ paraformaldehyde and visualized under fluorescence microscopy. S2 lacO cells with autosomal integration of lacO repeats were a gift from Patrick Heun. For luciferase assay, seven lac-operator repeat containing pGL3 (Promega) vector (pGL3-luc7lacO) was generated with amplified LacO-repeats from F42B2 cells, using primers with an integrated Nhel site. Integration of the seven LacO-repeats followed after digestion with Nhe1. Transfections for luciferase assay were done with $500 \mathrm{ng}$ of pGL3-luc-7lacO and $500 \mathrm{ng}$ of LacI-insulator fusion construct. $500 \mathrm{ng}$ of pSV/ $\beta$-Gal construct (Promega) was used as an internal control reporter. Cells were harvested and luciferase activity was measured in a Luminometer. LacZ was used for normalization.

Immunofluorescence staining and microscopy. After 48 hours of transfection, cells were collected and washed two times with PBS. Cells were fixed in $4 \%$ paraformaldehyde in PBS at room temperature (RT) for 15 to $20 \mathrm{~min}$. The fixative was removed and the cells were washed 3 times with PBS. Fixed cells were once washed with $0.5 \%$ TritonX-100 in PBS for 5 to 10 minutes. After 2 washes with PBS, cells were blocked with 5\% FCS at RT for $30 \mathrm{~min}$. Blocking solution was removed and the specimen was washed twice with PBS and primary antibody (anti-CP190-Bx63) in a ratio of $1: 4$ in PBS was added and incubated for at least 1 hour at RT. The sample was rinsed 3 times in PBS, each wash spanning $5 \mathrm{~min}$. The sample was incubated with fluorochrome-conjugated secondary antibody (anti-mouse HRP Red 568) in a ratio of $1: 200$ and allowed to incubate in dark for 1 hour at RT. The samples were rinsed as done after the primary antibody treatment, stained with Hoechst-33342 for 5 to $10 \mathrm{~min}$ at RT. After a wash with PBS, the specimens were dehydrated in $90 \%$ ethanol at RT for 2 to $3 \mathrm{~min}$ and mounted on slides with coverslips. Slides were analysed immediately or stored at $4^{\circ} \mathrm{C}$. All samples were analysed in Axio Observer Z1 fluorescence microscope (Zeiss).

1. Dixon, J. R. et al. Topological domains in mammalian genomes identified by analysis of chromatin interactions. Nature 485, 376-80 (2012).

2. Hou, C., Li, L., Qin, Z. S. \& Corces, V. G. Gene density, transcription, and insulators contribute to the partition of the Drosophila genome into physical domains. Mol Cell 48, 471-84 (2012).

3. Sexton, T. et al. Three-dimensional folding and functional organization principles of the Drosophila genome. Cell 148, 458-72 (2012).

4. Matharu, N. K., Hussain, T., Sankaranarayanan, R. \& Mishra, R. K. Vertebrate homologue of Drosophila GAGA factor. J Mol Biol 400, 434-47 (2010).

5. Wallace, J. A. \& Felsenfeld, G. We gather together: insulators and genome organization. Curr Opin Genet Dev 17, 400-7 (2007).

6. Gaszner, M., Vazquez, J. \& Schedl, P. The Zw5 protein, a component of the scs chromatin domain boundary, is able to block enhancer-promoter interaction. Genes Dev 13, 2098-107 (1999).

7. Gerasimova, T. I., Gdula, D. A., Gerasimov, D. V., Simonova, O. \& Corces, V. G. A Drosophila protein that imparts directionality on a chromatin insulator is an enhancer of position-effect variegation. Cell 82, 587-97 (1995).
8. Hart, C. M., Zhao, K. \& Laemmli, U. K. The scs' boundary element: characterization of boundary element-associated factors. Mol Cell Biol 17, 999-1009 (1997)

9. Moon, H. et al. CTCF is conserved from Drosophila to humans and confers enhancer blocking of the Fab-8 insulator. EMBO Rep 6, 165-70 (2005).

10. Schweinsberg, S. et al. The enhancer-blocking activity of the Fab-7 boundary from the Drosophila bithorax complex requires GAGA-factor-binding sites. Genetics 168, 1371-84 (2004).

11. Ahanger, S. H., Shouche, Y. S. \& Mishra, R. K. Functional sub-division of the Drosophila genome via chromatin looping: The emerging importance of CP190. Nucleus 4, 115-22 (2013).

12. Yang, J. \& Corces, V. G. Insulators, long-range interactions, and genome function. Curr Opin Genet Dev 22, 86-92 (2012).

13. Capelson, M. \& Corces, V. G. Boundary elements and nuclear organization. Biol Cell 96, 617-29 (2004).

14. West, A. G., Gaszner, M. \& Felsenfeld, G. Insulators: many functions, many mechanisms. Genes Dev 16, 271-88 (2002).

15. Dorman, E. R., Bushey, A. M. \& Corces, V. G. The role of insulator elements in large-scale chromatin structure in interphase. Semin Cell Dev Biol 18, 682-90 (2007).

16. Ishihara, K., Oshimura, M. \& Nakao, M. CTCF-dependent chromatin insulator is linked to epigenetic remodeling. Mol Cell 23, 733-42 (2006).

17. Li, M., Belozerov, V. E. \& Cai, H. N. Modulation of chromatin boundary activities by nucleosome-remodeling activities in Drosophila melanogaster. Mol Cell Biol 30, 1067-76 (2010).

18. Clapier, C. R. \& Cairns, B. R. The biology of chromatin remodeling complexes. Annu Rev Biochem 78, 273-304 (2009).

19. Cuddapah, S. et al. Global analysis of the insulator binding protein CTCF in chromatin barrier regions reveals demarcation of active and repressive domains. Genome Res 19, 24-32 (2009).

20. Fu, Y., Sinha, M., Peterson, C. L. \& Weng, Z. The insulator binding protein CTCF positions 20 nucleosomes around its binding sites across the human genome. PLoS Genet 4, e1000138 (2008).

21. Kanduri, M. et al. Multiple nucleosome positioning sites regulate the CTCFmediated insulator function of the $\mathrm{H} 19$ imprinting control region. Mol Cell Biol 22, 3339-44 (2002)

22. Bartkuhn, M. et al. Active promoters and insulators are marked by the centrosomal protein 190. EMBO J 28, 877-88 (2009).

23. Negre, N. et al. A comprehensive map of insulator elements for the Drosophila genome. PLoS Genet 6, e1000814 (2010).

24. Robinett, C. C. et al. In vivo localization of DNA sequences and visualization of large-scale chromatin organization using lac operator/repressor recognition. J Cell Biol 135, 1685-700 (1996)

25. Jegou, T. et al. Dynamics of telomeres and promyelocytic leukemia nuclear bodies in a telomerase-negative human cell line. Mol Biol Cell 20, 2070-82 (2009).

26. Kitchen, N. S. \& Schoenherr, C. J. Sumoylation modulates a domain in CTCF that activates transcription and decondenses chromatin. J Cell Biochem 111, 665-75 (2010).

27. Gerasimova, T. I., Lei, E. P., Bushey, A. M. \& Corces, V. G. Coordinated control of dCTCF and gypsy chromatin insulators in Drosophila. Mol Cell 28, 761-72 (2007).

28. Mohan, M. et al. The Drosophila insulator proteins CTCF and CP190 link enhancer blocking to body patterning. EMBO J 26, 4203-14 (2007).

29. Bushey, A. M., Ramos, E. \& Corces, V. G. Three subclasses of a Drosophila insulator show distinct and cell type-specific genomic distributions. Genes Dev 23, 1338-50 (2009).

30. Mendiburo, M. J., Padeken, J., Fulop, S., Schepers, A. \& Heun, P. Drosophila CENH3 is sufficient for centromere formation. Science 334, 686-90 (2011)

31. Gunther, K. et al. Differential roles for MBD2 and MBD3 at methylated CpG islands, active promoters and binding to exon sequences. Nucleic Acids Res 41, 3010-21 (2013)

32. Pirrotta, V. \& Li, H. B. A view of nuclear Polycomb bodies. Curr Opin Genet Dev 22, 101-9 (2012)

33. Belmont, A. S. \& Straight, A. F. In vivo visualization of chromosomes using lac operator-repressor binding. Trends Cell Biol 8, 121-4 (1998)

34. Chen, D., Belmont, A. S. \& Huang, S. Upstream binding factor association induces large-scale chromatin decondensation. Proc Natl Acad Sci U S A 101, 15106-11 (2004).

35. Deng, H. et al. Ectopic histone H3S10 phosphorylation causes chromatin structure remodeling in Drosophila. Development 135, 699-705 (2008).

36. Li, Y., Danzer, J. R., Alvarez, P., Belmont, A. S. \& Wallrath, L. L. Effects of tethering HP1 to euchromatic regions of the Drosophila genome. Development 130, 1817-24 (2003)

37. Tumbar, T., Sudlow, G. \& Belmont, A. S. Large-scale chromatin unfolding and remodeling induced by VP16 acidic activation domain. J Cell Biol 145, 1341-54 (1999).

38. Verschure, P. J. et al. In vivo HP1 targeting causes large-scale chromatin condensation and enhanced histone lysine methylation. Mol Cell Biol 25, 4552-64 (2005).

39. Schwartz, Y. B. et al. Nature and function of insulator protein binding sites in the Drosophila genome. Genome Res 22, 2188-98 (2012). 
40. Herold, M., Bartkuhn, M. \& Renkawitz, R. CTCF: insights into insulator function during development. Development 139, 1045-57 (2012).

41. Jenuwein, T. \& Allis, C. D. Translating the histone code. Science 293, 1074-80 (2001).

42. Fischle, W., Wang, Y. \& Allis, C. D. Histone and chromatin cross-talk. Curr Opin Cell Biol 15, 172-83 (2003).

43. West, A. G., Huang, S., Gaszner, M., Litt, M. D. \& Felsenfeld, G. Recruitment of histone modifications by USF proteins at a vertebrate barrier element. Mol Cell 16, 453-63 (2004).

44. Donze, D. \& Kamakaka, R. T. RNA polymerase III and RNA polymerase II promoter complexes are heterochromatin barriers in Saccharomyces cerevisiae. EMBO J 20, 520-31 (2001)

45. Donze, D. \& Kamakaka, R. T. Braking the silence: how heterochromatic gene repression is stopped in its tracks. Bioessays 24, 344-9 (2002).

46. Mito, Y., Henikoff, J. G. \& Henikoff, S. Histone replacement marks the boundaries of cis-regulatory domains. Science 315, 1408-11 (2007).

47. Zhao, H., Kim, A., Song, S. H. \& Dean, A. Enhancer blocking by chicken betaglobin $5^{\prime}$-HS4: role of enhancer strength and insulator nucleosome depletion. J Biol Chem 281, 30573-80 (2006).

48. Burke, L. J. et al. CTCF binding and higher order chromatin structure of the H19 locus are maintained in mitotic chromatin. EMBO J 24, 3291-300 (2005).

49. Baniahmad, A. et al. The tau 4 activation domain of the thyroid hormone receptor is required for release of a putative corepressor(s) necessary for transcriptional silencing. Mol Cell Biol 15, 76-86 (1995).

\section{Acknowledgments}

We would like to thank Nadine Müller for microscopic evaluation and Patrick Heun for the lacO/LacI system in Drosophila S2 cells and Drosophila vectors. Research in R.R. and Y.S.S. laboratories is supported by the Deutsche Forschungsgemeinschaft (DFG, TRR81), Germany and Department of Biotechnology (DBT), India, respectively. S.H.A.

acknowledges fellowships from Deutscher Akademischer Austausch Dienst (DAAD), Germany and Indian counsel of Medical Research (ICMR), India. The authors declare no conflict of interest.

\section{Author contributions}

S.H.A. designed and performed the experiments, analysed the data and wrote the paper. K.G. verified the expression of fusion constructs and their localization pattern in U2OS cells. O.W. performed the array experiments in S2 cells. M.B. designed and analysed the experiments. R.R.B. and Y.S.S. provided advice throughout the work. R.R. designed the experiments, analysed the data and gave advice on manuscript preparation. All authors reviewed the manuscript.

\section{Additional information}

Supplementary information accompanies this paper at http://www.nature.com/ scientificreports

Competing financial interests: The authors declare no competing financial interests.

How to cite this article: Ahanger, S.H. et al. Ectopically tethered CP190 induces large-scale chromatin decondensation. Sci. Rep. 4, 3917; DOI:10.1038/srep03917 (2014).

(c) (i) (-) $\Theta$ This work is licensed under a Creative Commons AttributionNonCommercial-NoDerivs 3.0 Unported license. To view a copy of this license, visit http://creativecommons.org/licenses/by-nc-nd/3.0 